Abanico Veterinario. Enero-Diciembre 2021; 11:1-10. http://dx.doi.org/10.21929/abavet2021.44 Caso Clínico. Recibido: 11/09/2021. Aceptado: 07/12/2021. Publicado: 28/12/2021. Clave: e2021-66. https://www.youtube.com/watch?v=P86RZLRDC1U

\title{
Inmunoterapia y manejo quirúrgico de Pythium insidiosum en un equino: reporte de caso
}

Surgical management and immunotherapy of Pythium insidiosum in a horse: case report Estrada-Coates Alejandro ${ }^{11 \mathrm{D} *}$, Alva-Trujillo Miriam ${ }^{1 \mathrm{ID}}$, Muñoz-Melgarejo Sergio ${ }^{1 \mathrm{ID}}$,
Canales-Rubio Miguel ${ }^{1 \mathrm{ID}}$, Herrera-Camacho José

${ }^{1}$ Facultad de Medicina Veterinaria y Zootecnia, Universidad Veracruzana. Veracruz, Ver., México. ${ }^{2}$ Instituto de Investigaciones Agropecuarias y Forestales-Universidad Michoacana de San Nicolás de Hidalgo. Hidalgo, México. ${ }^{*}$ Autor responsable y de correspondencia: Estrada Coates Alejandro. Facultad de Medicina Veterinaria y Zootecnia, Universidad Veracruzana. Miguel Ángel de Quevedo s/n esq. Yáñez. Col. Unidad Veracruzana. CP. 91710. Veracruz, Ver., México. E-mail: aestrada@uv.mx, mialva@uv.mx, smunoz@uv.mx,mcanales@uv.mx, jose.camacho@umich.mx

\section{RESUMEN}

La pitiosis cutánea equina es causada por el oomiceto Pythium insidiosum, el cual generalmente produce lesiones ulcerativas granulomatosas de progresión rápida en las partes distales de las extremidades y del abdomen. Es una enfermedad distribuida globalmente y con mayor prevalencia en regiones tropicales y subtropicales. El objetivo de este reporte de caso fue describir las lesiones granulomatosas, con tejido necrótico y exudado fibrinosanguinolento en la región palpebral del ojo izquierdo ocasionadas por Pythium insidiosum en una yegua cuarto de milla en el estado de Veracruz, México. El diagnóstico se fundamentó en las características clínicas de la lesión y la histopatología. Debido a la extensión de las lesiones, se realizó la resección quirúrgica en combinación con la inmunoterapia. El tratamiento con la inmunoterapia demostró ser una alternativa eficaz para lograr la resolución completa de las lesiones cutáneas.

Palabras clave: Pythium insidiosum, pitiosis, equino, inmunoterapia.

\begin{abstract}
Equine cutaneous pythiosis is caused by the oomycete Pythium insidiosum, which generally produces rapidly progressive granulomatous ulcerative lesions on the distal parts of the limbs and abdomen. It is a globally distributed disease with higher prevalence in tropical and subtropical regions. The objective of this case report was to describe granulomatous lesions with necrotic tissue and fibrinous-anginolous exudate in the palpebral region of the left eye caused by Pythium insidiosum in a quarter horse mare in the state of Veracruz, Mexico. The diagnosis was based on the clinical characteristics of the lesion and histopathology. Due to the extent of the lesions, surgical resection was performed in combination with immunotherapy. Treatment with immunotherapy proved to be an effective alternative to achieve complete resolution of the skin lesions.
\end{abstract}

Keywords: Pythium insidiosum, pythiosis, equine, immunotherapy. 


\section{INTRODUCCIÓN}

El agente etiológico Pythium insidiosum ( $P$. insidiosum) pertenece a la clase Oomycetes, cuyas zoosporas carecen de quitina y ergosterol en su composición celular, y causa una condición médica que se le conoce como pitiosis cutánea equina, la cual se presenta en regiones tropicales y subtropicales (Gaastra et al., 2010, Santos et al., 2011a, Cardona et al., 2013).

El equino es la especie con más casos notificados, aunque también hay reportes en cánidos (forma intestinal), felinos, animales silvestres, bovinos, ovinos (Loreto et al., 2014) y humanos (He et al., 2016, Chitasombat et al., 2020). Las lesiones cutáneas son más frecuentes en las partes distales de las extremidades, el abdomen ventral y lateral, la región pectoral y la boca, quizá por el mayor contacto de estas áreas con aguas potencialmente contaminadas con zoosporas. Las lesiones son ulcerativas granulomatosas, con bordes irregulares, trayectos fistulosos o cavitaciones y con presencia de masas necróticas duras que se desprenden fácilmente (Atiba et al., 2020, Souto et al., 2021).

El diagnóstico se basa primordialmente en la observación de las características clínicas de las lesiones cutáneas y la histopatología (Schanzembach et al., 2019). La resección quirúrgica de todo el tejido afectado, en combinación con un inmunoterapéutico que contiene antígenos de $P$. insidiosum, es uno de los tratamientos más eficaces que existen en la actualidad (Mendoza et al., 2003; Mendoza et al., 2004).

Este reporte se describe las características clínicas de un caso de pitiosis en un equino, con lesiones focalizadas en la región palpebral, su diagnóstico histopatológico y su tratamiento mediante inmunoterapia.

\section{DESCRIPCIÓN DEL CASO}

Una yegua Cuarto de milla, de 11 años, fue remitida al servicio ambulatorio del Hospital Veterinario para Grandes Especies de la Facultad de Medicina Veterinaria y Zootecnia de la Universidad Veracruzana, para evaluarle una lesión granulomatosa de crecimiento rápido y sin respuesta a tratamiento.

Durante la evaluación médica se pudo observar que la yegua presentaba una lesión ulcerativa granulomatosa, con tejido necrótico y exudado fibrinosanguinolento en la región palpebral del ojo izquierdo, de aproximadamente $20 \mathrm{~cm} \times 16 \mathrm{~cm}$ (Figura 1). Durante la palpación de la zona afectada se pudo evidenciar la presencia de trayectos fistulosos y exudado caseificado de color blanco amarillento. La yegua presentaba prurito intenso, dolor en la región afectada, mucosas pálidas y pérdida de peso progresiva. En la citometría hemática los hallazgos relevantes fueron anemia y leucocitosis por neutrofilia. 


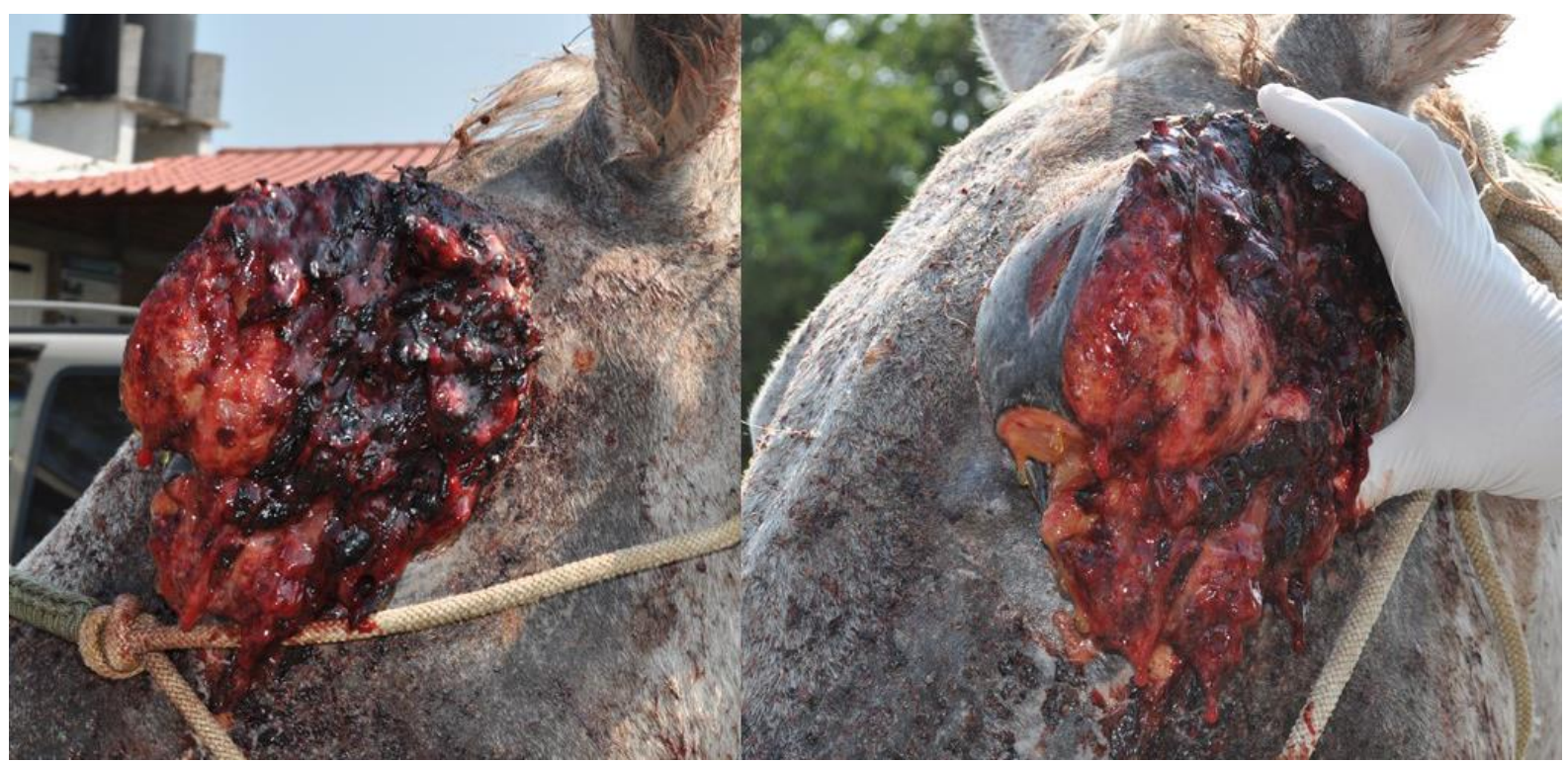

Figura 1. Lesión ulcerativa granulomatosa, con tejido necrótico y exudado fibrinosanguinolento en la región palpebral del ojo izquierdo

Se tomaron muestras de tejido, las cuales fueron conservadas en formol al 10\% para su posterior análisis histopatológico. En la evaluación histopatológica se observó un intenso infiltrado de abundantes eosinófilos, mastocitos reactivos, presencia de zonas de necrosis multifocal y fenómeno de Splendore-Hoeppli (Figura 2); de igual forma, se identificó la presencia de hifas ocasionalmente septadas, ramificadas y de pared lisa que coincidían con P. insidiousum (Figura 3).

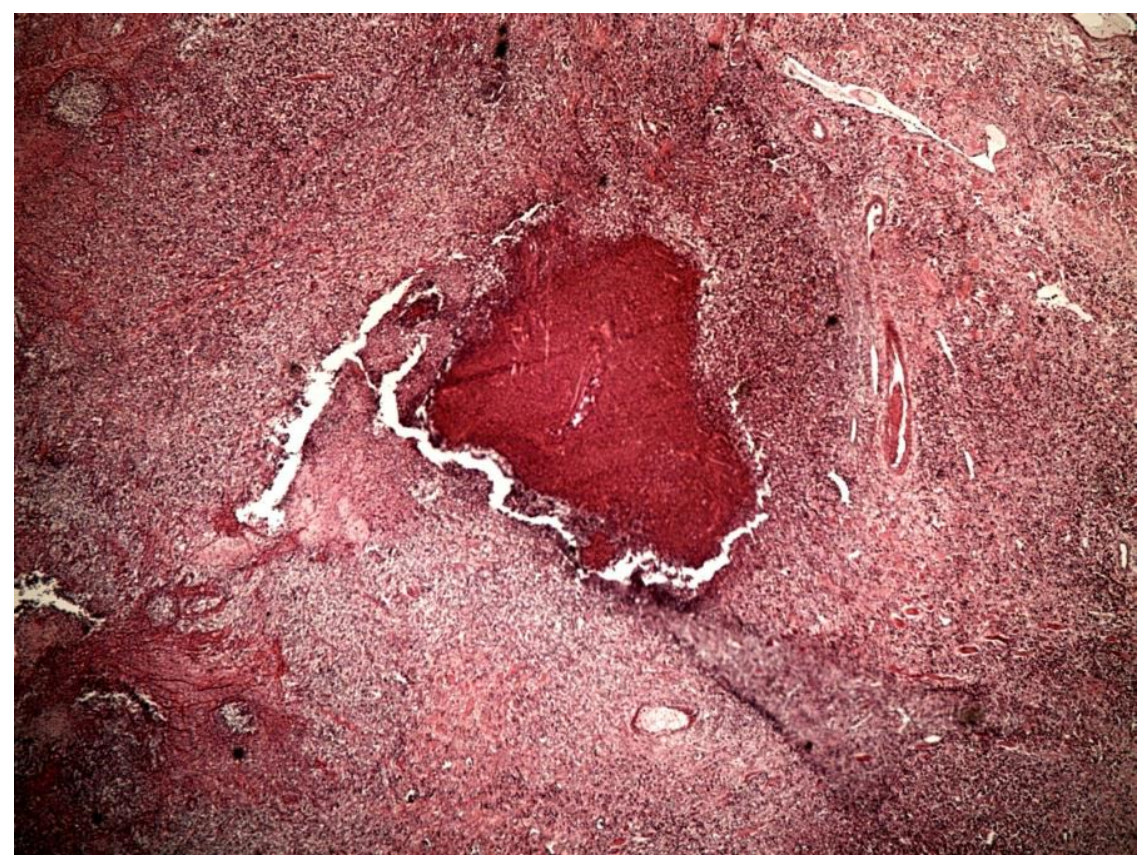

Figura 2. Fenómeno de Splendore-Hoeppli (tinción con hematoxilina y eosina, 40X) 


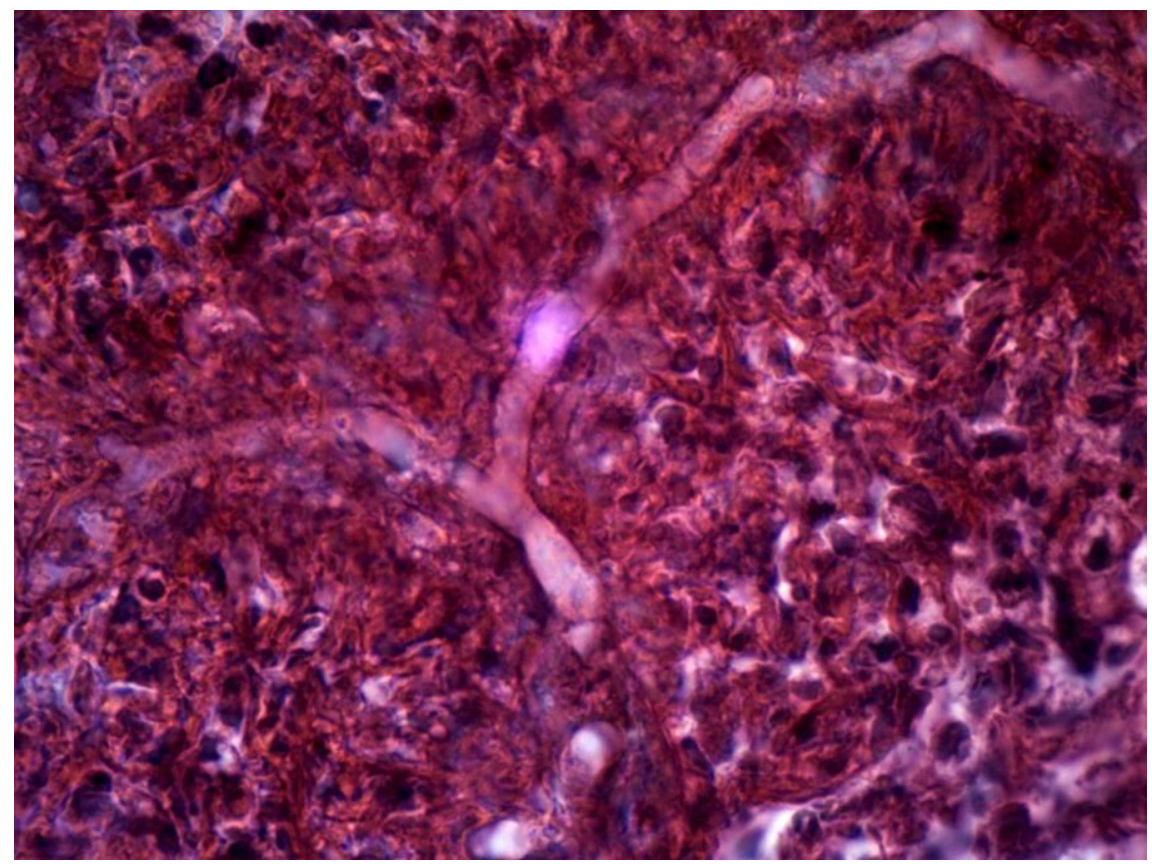

Figura 3. Hifas de Pythium insidiosum ramificadas y moderadamente septadas (tinción con hematoxilina y eosina, 100X)

Después de la evaluación clínica y los resultados histopatológicos, se removió el tejido afectado mediante resección quirúrgica con la yegua en estación. Se mantuvo a la yegua en ayuno 12 horas previo al procedimiento quirúrgico, se colocó un catéter de $14 \mathrm{G}$ en la vena yugular y se administró gentamicina $(6.6 \mathrm{mg} / \mathrm{kg} \mathrm{IV})$, flunixin de meglumine (1.1 $\mathrm{mg} / \mathrm{kg}$ IV) y vía intramuscular $22000 \mathrm{Ul} / \mathrm{kg}$ de penicilina procaínica.

Se mantuvo a la yegua sedada con un bolo inicial de $0.01 \mathrm{mg} / \mathrm{kg}$ de clorhidrato de detomidina y de mantenimiento con una infusión constante del mismo producto, a una dosis total de $10 \mathrm{mg}$ en $250 \mathrm{~mL}$ de solución salina a efecto $(0.04 \mathrm{mg} / \mathrm{mL})$, y se realizaron bloqueos regionales con clorhidrato de lidocaína al $2 \%$. El área afectada fue preparada con técnica aséptica con yodo povidona al $8 \%$ y agua estéril. Durante el procedimiento quirúrgico se pudo remover todo el tejido granulomatoso y las concreciones ("kunkers") y se evitó la enucleación del globo ocular.

Debido a la extensión de las lesiones y el riesgo de que existieran hifas en tejidos profundos, se decidió utilizar un inmunoterapéutico comercial que se ha desarrollado a partir de proteínas de las hifas y los metabolitos de $P$. insidiosum. El tratamiento consistió en aplicar $1 \mathrm{~mL}$ vía subcutánea en el tercio distal del cuello los días 0 (día de la cirugía), 7 y 21 después del procedimiento quirúrgico, de acuerdo con las especificaciones del fabricante (Pan American Veterinary Laboratories, Lexington, Texas $®$ ). Posterior a la inoculación del inmunoterapéutico, no se observaron efectos secundarios en la zona de aplicación. 
El tratamiento posquirúrgico consistió en un vendaje compresivo, con un patrón cruzado, para no interferir con el campo de visión del ojo contralateral y la limpieza de la herida se llevó a cabo utilizando solución isotónica cada 24 horas. Al mismo tiempo, se mantuvo en gentamicina $(6.6 \mathrm{mg} / \mathrm{kg} \mathrm{IV})$, penicilina procaínica $(22,000 \mathrm{UI} / \mathrm{kg} \mathrm{IM})$ y flunixin de meglumine $(1.1 \mathrm{mg} / \mathrm{kg} \mathrm{IV})$ durante cinco días. Durante el posoperatorio se monitoreó la fase de proliferación de la herida, para asegurar adecuada formación de tejido de granulación y apropiadas epitelización y remodelación del tejido.

\section{DISCUSIÓN}

La mayoria de los casos reportados en equinos son en Sudamérica (Salas et al., 201, Cardona et al., 2014, Dória et al., 2015, Schanzembach et al., 2019, Paz et al., 2021), lo cual se relaciona con sus regiones tropiales y subtropicales. El estado de Veracruz presenta condiciones ambientales similares, por ejemplo, abundantes lluvias que favorecen el estancamiento de agua en las praderas y temperaturas elevadas, que benefician al agente etiológico y su contacto con los equinos (Gaastra et al., 2010).

Las lesiones cutáneas encontradas en la region periorbital de la yegua concuerdan con la descripcion de las lesiones cutáneas granulomatosas descritas en otros informes de caso en diferentes países (Bezerra-Junior et al., 2010, Mosbah et al., 2012, Cardona et al., 2014, Tartor et al., 2020), en donde se reportan lesiones cutáneas en regiones anatómicas que se encuentran en contacto directo con aguas estancadas contaminadas con zoosporas de $P$. insidiosum, por lo que las lesiones en regiones altas de la cabeza no son comunes. Sin embargo, en Tailandia se notificó el primer caso con lesiones de pitiosis dentro de la cavidad nasal de una yegua gestante y anticuerpos para $P$. insidisum en tres caballos (Tonpitak et al., 2018, Mar Htun et al., 2021).

El diagnóstico de pitiosis se basa fundamentalmente en la observación de las características clínicas de las lesiones cutáneas. De igual forma, es importante establecer los diagnósticos diferenciales de estas lesiones, ya que a menudo se diagnostica erróneamente como habronemiasis cutánea, tejido de granulación exuberante o sarcoides, así que es importante llevar a cabo estudios histopatológicos para el diagnósitico definitivo.

Los hallazgos histopatológicos de este caso concuerdan con lo descrito por Márquez et al. (2010), donde se pudo obervar el fenómeno de Splendore-Hoeppli y la identificación de hifas septadas. Este fenómeno se considera una estrategia adaptativa del agente etiológico con el objetivo de asegurar su proliferación y sobrevivencia en el tejido del hospedero, lo cual se puede confirmar por la presencia de hifas viables dentro de la reacción eosinofílica (Martins et al., 2012).

El tratamiento de elección para $P$. insidiosum sigue siendo la resección quirúrgica de todo el tejido afectado. Sin embargo, algunos estudios han demostrado que la combinación de 
la resección quirúrgica y antibióticos sistémicos como la anfotericina $\mathrm{B}$ y el miconazol, administrados vía perfusión regional en las extremidades afectadas, obtuvo una resolución del 92\% (Worster at al., 2000, Dória et al., 2012). En contraste, Pires et al. (2013) solamente obtuvieron un $60 \%$ de resolución de las lesiones cutáneas de pitiosis con la anfotericina B. Este tipo de combinación de terapias se lleva a cabo cuando la lesión ha invadido otro tipo de estructuras anatómicas, como músculos, tendones y huesos, que no pueden ser removidas quirúrgicamente.

En caso de que la resección quirúrgica sea parcial debido a la profundidad de la lesión, se puede utilizar inmunoterapia a base de proteínas de las hifas de $P$. insidiosum, la cual ha tenido una eficacia del $90 \%$ en casos de lesiones agudas menores a 60 días y del $20 \%$ en casos crónicos que superan los dos meses (Mendoza et al., 2003, Mendoza et al., 2005). La respuesta inmunológica fue similar a lo reportado por Pereira et al. (2011), en donde a los siete días de haber inoculado la primera dosis del inmunoterapéutico, la yegua ya no presentó evidencia de lesiones por $P$. insidiosum ni prurito e inició la fase de remodelación de la herida (Figura 4).

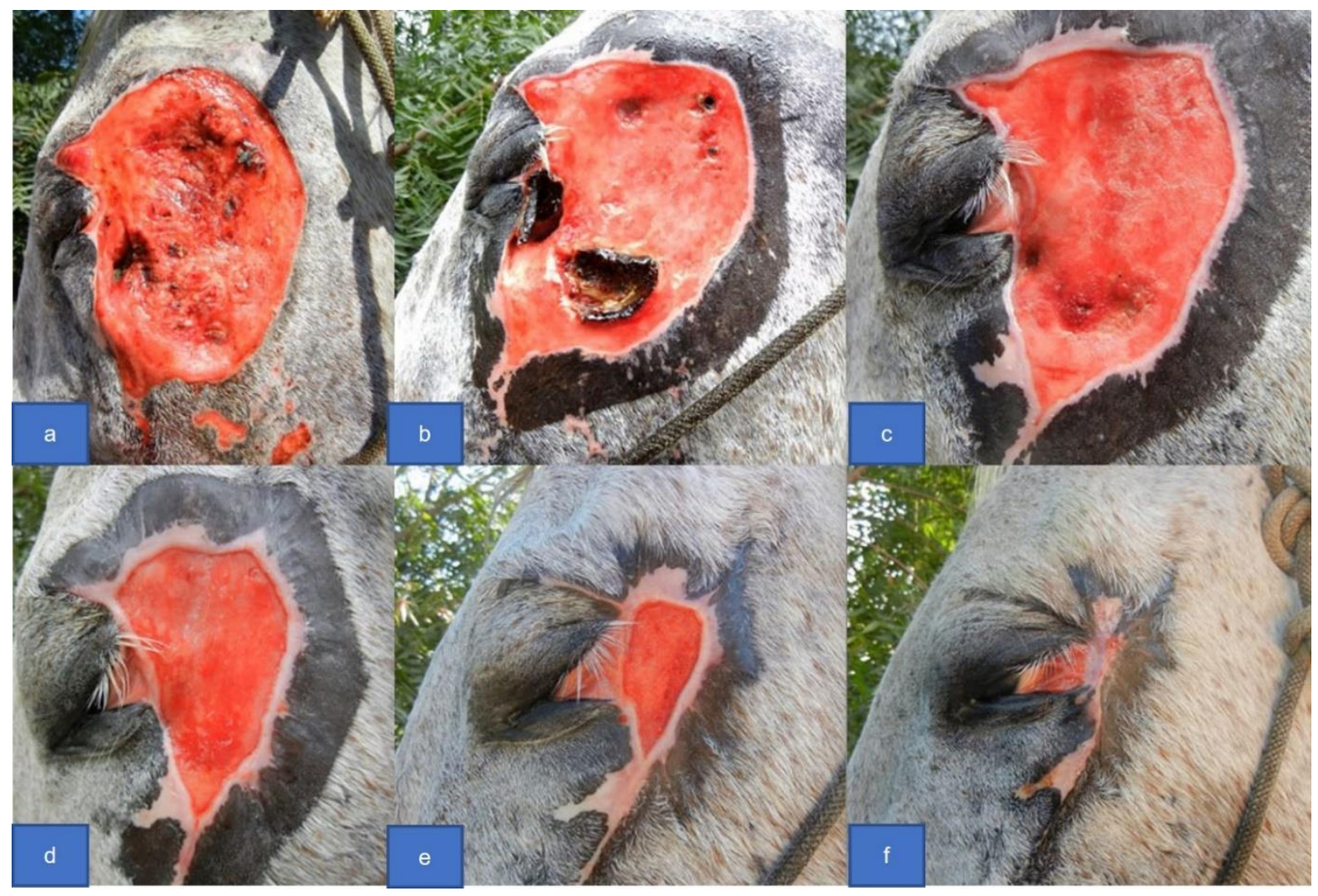

Figura 4. Fase inicial, proliferación y remodelación. (a) Lesión postquirúrgica inicial; (b-f) las imágenes muestran las fases de proliferación y remodelación. Al final del tratamiento solo se aprecia una lesión cicatricial con resolución completa del caso 
El éxito de la inmunoterapia se basa en que propicia el cambio de una respuesta mediada por anticuerpos a una mediada por células. Normalmente, predomina una respuesta regulada por linfocitos colaboradores tipo 2 (Th2), con liberación de interleucinas (IL) 4 y 5 y activación de eosinófilos y mastocitos, que resulta en una inflamación eosinofílica. En contraparte, la inmunoterapia favorece una respuesta regulada por linfocitos colaboradores tipo 1 (Th1), con liberación de IL-2 e IFN-y y activación de linfocitos T y macrófagos, quienes destruyen a $P$. insidiosum (Loreto et al., 2014). A pesar de las propiedades curativas de la inmunoterapia, Santos et al. (2011b) reportaron que probablemente los anticuerpos que se producen no son suficientes para prevenir la reinfección a corto y largo plazo.

\section{CONCLUSIÓN}

El resultado exitoso de los tratamientos para la pitiosis cutánea depende fundamentalmente de un rápido diagnóstico y del tratamiento temprano, con terapias combinadas para tener un buen pronóstico. Este es el primer reporte de caso que documenta el diagnóstico de $P$. insidiousum en equinos en el estado de Veracruz.

\section{LITERATURA CITADA}

ATIBA A, Ghazy A, Hamad MH. 2020. Evaluating the efficacy of surgical excision and topical dimethyl sulphoxide (DMSO) in the treatment of equine cutaneous pythiosis. Iranian Journal of Veterinary Research. 21(4):301-307. ISSN: 2252-0589 PMC7871735 https://www.ncbi.nlm.nih.gov/pmc/articles/PMC7871735/

BEZERRA-JUNIOR P, Pedroso P, Pavarini S, Dalto A, Santurio J, Driemeier D. 2010. Equine intestinal pythiosis in Southern Brazil. Arquivo Brasileiro de Medicina Veterinária e Zootecnia. 62(2):481-483. ISSN: 1678-4162. https://doi.org/10.1590/S010209352010000200031

CARDONA JA, Vargas-Viloria M, Perdomo S. 2013. Pitiose cutânea em equinos: uma revisão. Revista CES Medicina Veterinaria y Zootecnia. 8(1):58-67. ISSN: 1900-9607. http://www.redalyc.org/articulo.oa?id=321428109005

CARDONA JA, Vargas-Viloria M, Perdomo S. 2014. Frecuencia de pythiosis cutánea en caballos de producción en explotaciones ganaderas de córdoba, Colombia. Revista de la Facultad de Medicina Veterinaria y de Zootecnia. 61(1):31-43. ISSN: 0120-2952. http://dx.doi.org/10.15446/rfmvz.v61n1.43882 
CHITASOMBAT MN, Jongkhajornpong P, Lekhanont K, Krajaejun T. 2020. Recent update in diagnosis and treatment of human pythiosis. Peer J. 8:e8555. ISSN:2167-8359. https://doi.org/10.7717/peerj.8555

DÓRIA RG, Carvalho MB, Freitas SH, Laskoski LM, Colodel EM, Mendonça FS, Silva M A, Grigoletto R, Fantinato NP. 2015. Evaluation of intravenous regional perfusion with amphotericin B and dimethylsulfoxide to treat horses for pythiosis of a limb. BMC Veterinary Research. 11:152. ISSN: 1746:6148. https://doi.org/10.1186/s12917-0150472-z

GAASTRA W, Lipman LJ, De Cock AW, Exel TK, Pegge RB, Scheurwater J, Vilela R, Mendoza L. 2010. Pythium insidiosum: an overview. Veterinary Microbiology. 146(1-2):116. ISSN: 1873-2542. https://doi.org/10.1016/j.vetmic.2010.07.019

HE H, Liu H, Chen X, Wu J, He M, Zhong X. 2016. Diagnosis and Treatment of Pythium Insidiosum Corneal Ulcer in a Chinese Child: A Case Report and Literature Review. The American Journal of Case Reports. 17:982-988. ISSN: 1941-5923.

https://doi.org/10.12659/ajcr.901158

LORETO ÉS, Tondolo JSM, Zanette RA, Alves SH, Santurio JM. 2014. Update on pythiosis immunobiology and immunotherapy. World Journal of Immunology. 4(2):88-97. ISSN: 2219-2824. http://dx.doi.org/10.5411/wji.v4.i2.88

MAR HTUN Z, Laikul A, Pathomsakulwong W, Yurayart C, Lohnoo T, Yingyong W, Kumsang $Y$, Payattikul $P$, Sae-Chew $P$, Rujirawat $T$, Jaturapaktrarak $C$, Chongtrakool $P$, Krajaejun T. 2021. An initial survey of 150 horses from Thailand for anti-Pythium insidiosum antibodies. Journal de Mycologie Medicale. 31(1): 101085. ISSN: 1773-0449. https://doi.org/10.1016/j.mycmed.2020.101085

MÁRQUEZ A, Salas Y, Canelón J, Perazzo Y, Colme-Nárez V. 2010. Descripción anatomopatológica de pitiosis cutánea en equinos. Revista de la Facultad de Ciencias Veterinarias UCV. 51(1):7-42. ISSN: 0258-6576.

https://www.redalyc.org/articulo.oa?id=373139075005

MARTINS TB, Kommers, GD, Trost ME, Inkelmann MA, Fighera RA, Schild AL. 2012. A comparative study of the histopathology and immunohistochemistry of pythiosis in horses, dogs and cattle. Journal of Comparative Pathology. 146(2-3):122-131. ISSN: 1532-3129. https://doi.org/10.1016/j.jcpa.2011.06.006 
MENDOZA L, Newton JC. 2005. Immunology and immunotherapy of the infections caused by Pythium insidiosum. Medical Mycology. 43(6):477-486. ISSN: 1460-2709. https://doi.org/10.1080/13693780500279882

MENDOZA L, Mandy W, Glass R. 2003. An improved Pythium insidiosum-vaccine formulation with enhanced immunotherapeutic properties in horses and dogs with pythiosis. Vaccine. 21(21-22):2797-2804. ISSN:1873-2518.

https://doi.org/10.1016/s0264-410x(03)00225-1

MENDOZA L, Prasla SH, Ajello L. 2004. Orbital pythiosis: a non-fungal disease mimicking orbital mycotic infections, with a retrospective review of the literature. Mycoses. 47(12):14-23. ISSN: 1439-0507 https://doi.org/10.1046/j.1439-0507.2003.00950.x

MOSBAH E, Karrouf G, Younis E, Saad H, Ahdy A, Zaghloul A. 2012. Diagnosis and surgical management of pythiosis in draft horses: Re-port of 33 cases in Egypt. Journal of Equine Veterinary Science. 32(3):164-169. ISSN: 0737-0806.

https://doi.org/10.1016/j.jevs.2011.08.014

PAZ G, Camargo GG, Cury JE, Apolonio E, Garces HG, Prado A, Chechi JL, Oliveira A L, Watanabe MJ, Bagagli E, Bosco S. 2021. Outbreak of equine pythiosis in a southeastern region of Brazil: Environmental isolation and phylogeny. Transboundary and Emerging Diseases. 10.1111/tbed.14135. ISSN: 1865-1682.

https://doi.org/10.1111/tbed.14135

PEREIRA C, Soares R, Santurio J, Marques L. 2011 Eficácia da imunoterapia no tratamento de pitiose facial em equino. Acta Scientiae Veterinariae. 39(1):955. ISSN: 1679-9216. http://www.ufrgs.br/actavet/39-1/PUB\%20955.pdf

PIRES L, Bosco S, da Silva NF, Jr, Kurachi C. 2013. Photodynamic therapy for pythiosis. Veterinary Dermatology. 24(1): 130-6.e30. ISSN: 1365-3164.

https://doi.org/10.1111/j.1365-3164.2012.01112.x

SALAS, Y, Márquez A, Canelón J, Perazzo Y, Colmenárez V, López JA. 2012. Equine pythiosis: report in crossed bred (Criole Venezuelan) horses. Mycopathologia. 174(56):511-517. ISSN: 1573-0832. https://doi.org/10.1007/s11046-012-9562-7

SANTOS C, Santurio J, Marques C. 2011a. Pitiose em animais de produção no Pantanal Mato-grossense. Pesquisa Veterinária Brasileira. 31(12):1083-89. ISSN:1678-5150. https://doi.org/10.1590/S0100-736X2011001200008 
SANTOS CE, Marques LC, Zanette RA, Jesus FP, Santurio JM. 2011b. Does immunotherapy protect equines from reinfection by the oomycete Pythium insidiosum? Clinical and Vaccine Immunology. 18(8):1397-1399. ISSN: 1556-679X. https://doi.org/10.1128/CVI.05150-11

SCHANZEMBACH M, Brayer D, Sallis S,César D, Matto C, Almeida R, Nan F, Rodríguez V, Parodi P, Pereira M, Gianneechini R, Rivero R. 2019. Descripción de un caso de pitiosis cutánea equina y su diagnóstico mediante diversas técnicas. Veterinaria (Montevideo). 55(212):96-101. ISSN: 0376-4362. http://dx.doi.org/10.29155/vet.55.212.8.

SOUTO EPF, Maia LA, Neto EMN, Kommers GD, Junior FG, Riet-Correa F, Galiza GJN, Dantas AFM. 2021. Pythiosis in equidae in Northeastern Brazil: 1985-2020. Journal of Equine Veterinary Science. 105. 103726. ISSN: 0737-0806.

https://doi.org/10.1016/j.jevs.2021.103726

TARTOR YH, Hamad MH, Abouzeid NZ, El-Belkemy FA. 2020. Equine pythiosis in Egypt: clinicopathological findings, detection, identification and genotyping of Pythium insidiosum. Veterinary Dermatology. 31(4):298-e73. ISSN:1365-3164. https://doi.org/10.1111/vde.12845

TONPITAK W, Pathomsakulwong W, Sornklien C, Krajaejun T, Wutthiwithayaphong S. 2018. First confirmed case of nasal pythiosis in a horse in Thailand. JMM Case Reports. 5(1) e005136. ISSN: 2053-3721. https://doi.org/10.1099/jmmcr.0.005136

WORSTER AA, Lillich JD, Cox JH, Rush BR. 2000. Pythiosis with bone lesions in a pregnant mare. Journal of the American Veterinary Medical Association. 216(11):17951760. ISSN: 0003-1488. https://doi.org/10.2460/javma.2000.216.1795 